\title{
Going through the process
}

\author{
Good practices and new initiatives increase the efficiency of the review process and the accessibility of \\ peer-reviewed research.
}

T

he feeling of hope, anticipation, accomplishment and even relief that is associated with submitting a manuscript is unique. Many authors see manuscript submission as the end of the process and hope for a quick and painless review and speedy publication that allows them to move on to the next stage. Most of the time, however, passing review can be a lengthy endeavor. In addition, the requirements and standards for publication are constantly changing in response to evolving demands imposed by the nature of the experimental data, expectations from the community or the needs of clinical applications. Here we provide a brief 'how to' guide for a smooth and efficient review and publication process in the context of current editorial practices at Nature Immunology.

At submission, it is good practice that the first authors and the corresponding authors be available to respond to any requests or correspondence from the editors. We will normally contact you within a week. The median time from submission to editorial decision at Nature Immunology was 4 days for the past 2 years. For manuscripts selected for peer review, we ask authors to supply a reporting checklist that is designed to improve the accuracy of experimental reporting and help the referees in their assessment (https://www.nature.com/ articles/ni.2603). The completed form should be sent to us within 48 hours of the notification that the manuscript is being peer reviewed. If authors are unavailable during this timeframe, we suggest including the Reporting Summary checklist (https:// www.nature.com/documents/nr-reportingsummary.pdf) and Editorial Policy Checklist (https://www.nature.com/documents/ nr-editorial-policy-checklist.pdf) with the original manuscript submission to avoid delays in the review process, as the referees are not queried until the forms are submitted.

For manuscripts that include large data sets, it is our policy that data be deposited in public repositories (https://www.nature. $\mathrm{com} /$ nature-research/editorial-policies/ reporting-standards) and that all accession codes and confidential reviewer passwords be provided at the time of submission in the cover letter, the Methods section and the Reporting Summary. This also applies to any previously unreported custom computer code or algorithm that is used in the study and is central to its main claims or is essential for evaluation of the experimental data and the conclusions. The provision of these data is needed for fair peer review.

After peer review, the editors will send a decision letter in which it should be clear if a resubmission is invited. Because manuscripts are rejected on the basis of a variety of criteria, including novelty and broad interest, simply addressing the technical aspects of the review might not address other critical points behind the original decision. We advise against revising and resubmitting manuscripts that have been rejected without consulting the editor in the form of an appeal.

Appeals after peer review should include a full point-by-point rebuttal letter. It is useful to address all points raised by the referees with detailed documentation and specific arguments, references to the literature to support claims of novelty and impact, and proposals of experimental plans or inclusion of existing data from the laboratory that can clarify or support the conclusions or interpretations. A useful appeal provides the same level of specificity and detail as the referees' comments. Editors cannot reverse decisions solely on the basis of opinion-driven or emotional appeals.
Before formal acceptance, manuscripts at Nature Immunology are developmentally edited by the editors to improve readability and accuracy of reporting, while figures and additional material need to be formatted according to the journal standards and style. Effective immediately, Nature Immunology is introducing Extended Data (https:// www.nature.com/documents/nature-papercomposition.pdf), which can include up to ten figures, is appended to the online PDF and must be cited in the main text. Any additional files, including supplementary notes or methods, equations, computational data, large tables and spreadsheets, and video or audio files, should be formatted as Supplementary Information. Source Data, which are full-length, unprocessed blots and gels and the raw data for all figures, should be provided as separate files that will be published online.

In terms of new editorial developments, Nature Immunology will soon be undertaking a trial of transparent peer review, in which the anonymous referees' reports and the author responses to these reports can be published online along with the paper, if the authors choose so. This option will be available after the referees have endorsed publication. The referees will be informed of the transparent review process during referee query. This trial, along with other policies currently in the pipeline, is a reflection of efforts at Springer Nature to increase the transparency of the review process and the reproducibility of the research we publish. Feedback from authors and the community about this trial and other editorial initiatives is welcomed, to help shape a process that is rigorous, fair and transparent.

Published online: 22 October 2019 https://doi.org/10.1038/s41590-019-0538-3 